\title{
Clinical Implications of Local Field Potentials for Understanding and Treating Movement Disorders
}

\author{
John A. Thompson ${ }^{a}$ David Lanctin ${ }^{b}$ Nuri Firat Ince ${ }^{c}$ Aviva Abosch ${ }^{a}$ \\ ${ }^{a}$ Department of Neurosurgery, University of Colorado School of Medicine, Aurora, Colo., ${ }^{b}$ Department of \\ Neurosurgery, Division of Epidemiology, School of Public Health, University of Minnesota, Minneapolis, Minn., and \\ 'Department of Biomedical Engineering, University of Houston, Houston, Tex., USA
}

\section{Key Words}

Local field potentials · Deep brain stimulation · Parkinson's disease $\cdot$ Essential tremor $\cdot$ Dystonia

\begin{abstract}
Background: Deep brain stimulation (DBS) for the treatment of movement disorders has provided researchers with an opportunity to record electrical oscillatory activity from electrodes implanted in deep brain structures. Extracellular activity recorded from a population of neurons, termed local field potentials (LFPs), has shed light on the pathophysiology of movement disorders and holds the potential to lead to refinement in existing treatments. Objective: This paper reviews the clinical significance of LFPs recorded from macroelectrodes implanted in basal ganglia and thalamic targets for the treatment of Parkinson's disease, essential tremor and dystonia. Methods: Neural population dynamics and subthreshold events, which are undetectable by single-unit recordings, can be examined with frequency band analysis of LFPs (frequency range: 1-250 Hz). Results: Of clinical relevance, reliable correlations between motor symptoms and components of the LFP power spectrum suggest that LFPs may serve as a biomarker for movement disorders. In particular, Parkinson's rigidity has been shown to correlate with
\end{abstract}

the power of beta oscillations $(13-30 \mathrm{~Hz})$, and essential tremor coheres with oscillations of 8-27 Hz. Furthermore, evidence indicates that the optimal contacts for DBS programming can be predicted from the anatomic location of beta and gamma bands $(48-200 \mathrm{~Hz})$. Conclusion: LFP analysis has implications for improved electrode targeting and the development of a real-time, individualized, 'closed-loop' stimulation system.

(c) 2014 S. Karger AG, Basel

\section{Introduction}

Over the past decade, deep brain stimulation (DBS) has largely replaced ablative techniques in the surgical treatment of movement disorders such as Parkinson's disease (PD), essential tremor (ET) and dystonia. In addition to providing critical symptomatic relief for patients, DBS provides a unique opportunity to record electrical oscillatory activity from deep brain structures. These oscillations, considered to represent aggregate neuronal discharge from neurons surrounding the electrode $[1,2]$, are referred to as local field potentials (LFPs).

DBS surgery involves the stereotactic implantation of a macroelectrode into a predetermined target region that 
is selected based on the indication being treated. Target coordinates are derived based on preoperative stereotactic imaging and verified or modified through intraoperative electrophysiological techniques [1]. The most commonly employed electrophysiological techniques include microelectrode recording and/or macrostimulation through the DBS electrode. Microelectrode recording allows the identification of single cells that are characteristic of the target of interest through firing rate, amplitude and activity pattern. For example, through microelectrode recordings, the subthalamic nucleus (STN) is usually identified by high background, increased firing rate and frequency of bursting neurons. The DBS electrode, which typically consists of four platinum-iridium contacts, is subsequently implanted based on the microelectrode findings. Both the microelectrode and DBS electrode are both capable of recording LFPs.

At present, DBS targets for movement disorders include the ventral intermediate nucleus of the thalamus for the treatment of ET, the STN or globus pallidus pars internus (GPi) for PD and the GPi for dystonia. Additionally, the pedunculopontine nucleus (PPN) is currently under investigation for the treatment of postural instability associated with PD $[3,4]$.

The following review focuses on the clinical significance of LFPs recorded in the context of surgery for movement disorders. Potential clinical applications of these data include the use of LFPs for the following purposes: (1) as a symptom biomarker, (2) to improve surgical targeting during DBS electrode implantation and (3) to inform a 'closed-loop' therapy device.

\section{Functional Characterization of LFPs}

LFPs are composite signals divided into a number of frequency bands, as follows: $0-3 \mathrm{~Hz}$ (delta), $4-7 \mathrm{~Hz}$ (theta), $8-12 \mathrm{~Hz}$ (alpha), $13-30 \mathrm{~Hz}$ (beta), 31-200 Hz (gam$\mathrm{ma}$ ), and $>200 \mathrm{~Hz}$ (high frequency; table 1). It should be noted that there is nothing inherently pathological about a given oscillatory frequency range. Rather, the frequency spectrum represents a complex ensemble of neuronal activity - the significance of which depends on its functional and spatial context. This complexity necessitates a thorough evaluation of LFPs for each DBS indication and for each electrode target location.

The instantaneous amplitude and power of the LFP recordings are believed to represent the degree of synchronization between neurons surrounding the electrode tip $[5,6]$. A transient increase in power, in response to a
Table 1. LFP frequency-based classification system

\begin{tabular}{lc}
\hline Frequency band & Range, $\mathrm{Hz}$ \\
\hline Delta & $0-3$ \\
Theta & $4-7$ \\
Alpha & $8-12$ \\
Beta & $13-30$ \\
Gamma & $31-200$ \\
High frequency & $>200 \mathrm{~Hz}$ \\
\hline
\end{tabular}

specific cue, is often referred to as an event-related synchronization (ERS), while a transient decrease in power is termed event-related desynchronization (ERD) [7]. ERD and ERS are typically calculated by averaging the power across time segments and comparing this average to a reference epoch [8]. ERD and ERS were initially thought to represent activation and inactivation in neuronal circuits, respectively. However, this theory is now disputed. For instance, ERDs detected in the alpha and beta frequency ranges in electroencephalograms were initially thought to represent an activated neural circuit. However, observations in electrocorticography experiments indicated that these cortical ERDs in the alpha and beta bands were associated with an ERS in the gammafrequency band. Therefore, an ERS can also be activating. Consequently, the interpretation of ERD and ERS phenomena is likely to depend on the tissue of interest and other components of the frequency band [9].

\section{LFP and Parkinson's Disease}

\section{Beta Oscillations Positively Correlate with}

Bradykinesia and Rigidity

In the context of PD, beta-band LFPs have received the most attention. This interest stems from observations in a 1-methyl-4-phenyl-1,2,3,6-tetrahydropyridine (MPTP)-treated primate model. The resulting death of dopaminergic neurons in the substantia nigra pars compacta, the hallmark of $\mathrm{PD}$, is characterized by the onset of Parkinson's motor symptoms such as rigidity and bradykinesia in these MPTP-treated monkeys [10-12]. Singleunit recordings from the GPi and the STN in these monkeys have documented an increased firing rate accompanied with synchronous oscillatory bursting activity that is not found in control subjects $[13,14]$.

The increased oscillatory activity observed in MPTPtreated monkeys was subsequently found in the beta-fre- 


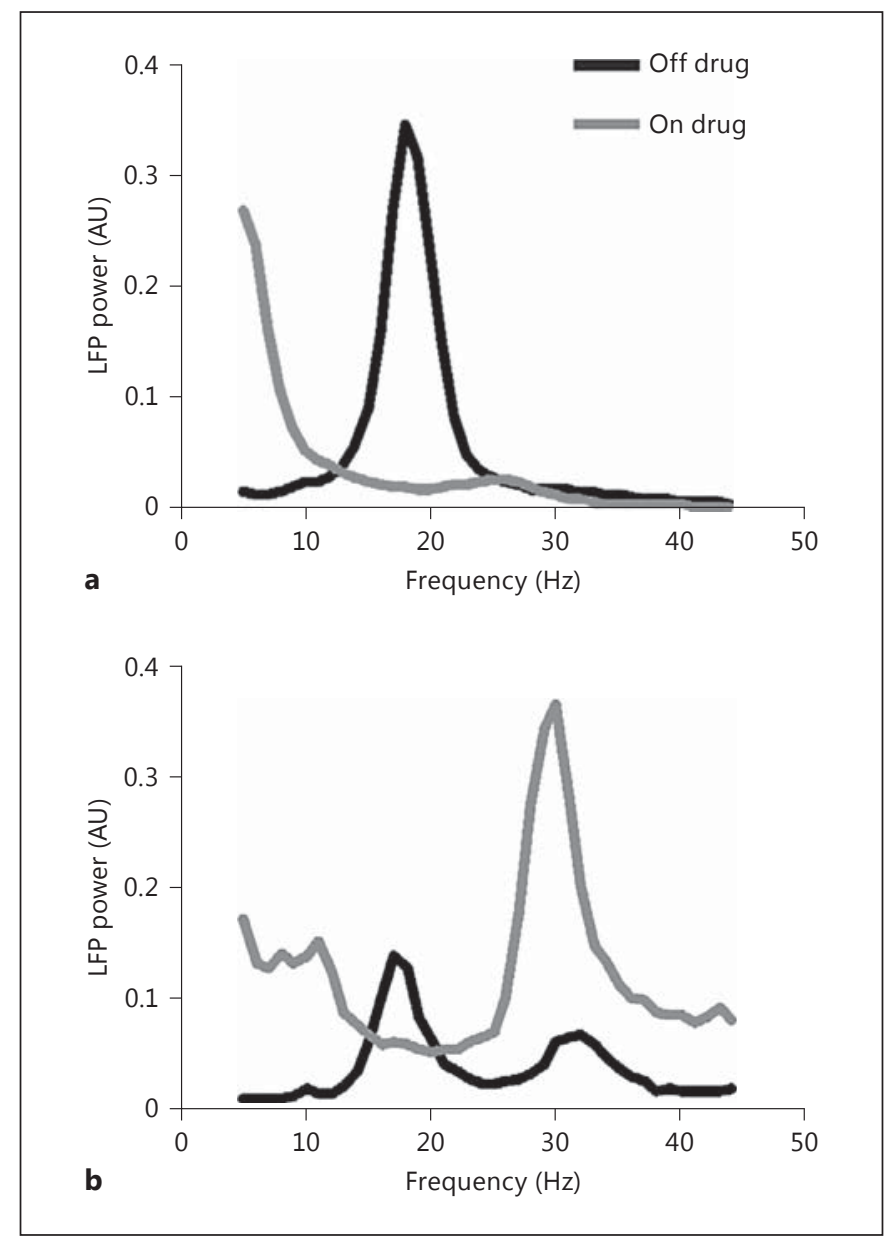

Fig. 1. LFP power spectra recorded from 2 patients with implanted STN electrodes for the treatment of PD. a The power of beta-frequency oscillations is severely diminished by dopaminergic medication in this patient. $\mathbf{b}$ A decrease in low beta oscillations (approx. $17 \mathrm{~Hz}$ ) is coupled with an increase in high beta oscillations (approx. $30 \mathrm{~Hz}$ ) in this patient. Taken with permission from Ray et al. [20].

quency band of LFPs recorded from the STN of human PD patients undergoing DBS surgery. Interestingly, the presence of this beta band is abolished by dopamine treatment (fig. 1) [15-19], and the degree of improvement in bradykinesia and rigidity following dopamine therapy has been shown to correlate with the magnitude of betaband suppression [20-22]. Conversely, Kuhn et al. [23] observed that excessive synchronous beta activity can be induced in patients undergoing GPi-targeted DBS for dystonia by using the dopamine antagonist tetrabenazine. This evidence suggests that highly synchronous beta activity is caused by dopamine depletion. Interestingly, initial studies did not find a correlation between raw beta

Clinical Correlations and Implications of LFPs power and severity of bradykinesia/rigidity in patients withdrawn from dopaminergic medication $[20,22,24$, 25]. However, the absence of LFP power normalization in these studies may reduce the ability to detect a significant correlation between beta oscillations and motor impairment. For example, beta power is known to vary spatially over small distances [26, 27]. Therefore, subtle differences in electrode location relative to target may decrease the correlation between motor impairment and raw beta power. More recent studies employing indirect strategies to normalize synchronous beta activity between subjects have documented a significant correlation between beta oscillations and rigidity and bradykinesia [25, 28, 29]. These normalization strategies include analysis of beta LFP signal complexity and the extent of phase coherence between adjacent macroelectrode contacts.

Similar to the effect of dopamine, STN-targeted DBS causes a suppression in beta-frequency oscillations, and the degree of improvement in rigidity and bradykinesia correlates with the magnitude of beta suppression (measured immediately after stimulus discontinuation) [20]; however, this has not been a universal finding [30]. A limitation of these studies is that the authors were not able to record LFP during stimulation. To address this, Rossi et al. [31] developed a stimulus suppressor capable of recording artifact-free beta-band LFPs while simultaneously stimulating the STN. Using this technique, these authors found that stimulation through the DBS electrode suppressed beta synchronization in patients who were off dopaminergic medication but not while they were on medication. Therefore, while DBS and dopaminergic medication both diminish beta-LFP power, the effect of dopaminergic medication appears to be stronger [32] (fig. 2).

Another line of evidence supporting the hypothesis that beta activity is antikinetic stems from studies correlating beta power with voluntary movement. A beta ERD was observed during movement initiation and ERS during movement termination [16, 33-35]. Moreover, when patients were cued not to move following movement preparation, a significant synchronization was observed in the beta-frequency band $[20-22,36]$. These observations support the hypothesis that beta-band activity is antikinetic.

While beta-band LFP activity has received intensive scrutiny in recent years, it is important to keep in mind that the boundaries of the LFP frequency bands are somewhat arbitrary. For instance, there is evidence that low beta activity $(12-20 \mathrm{~Hz})$ may derive from a different underlying physiological process than high beta activity 


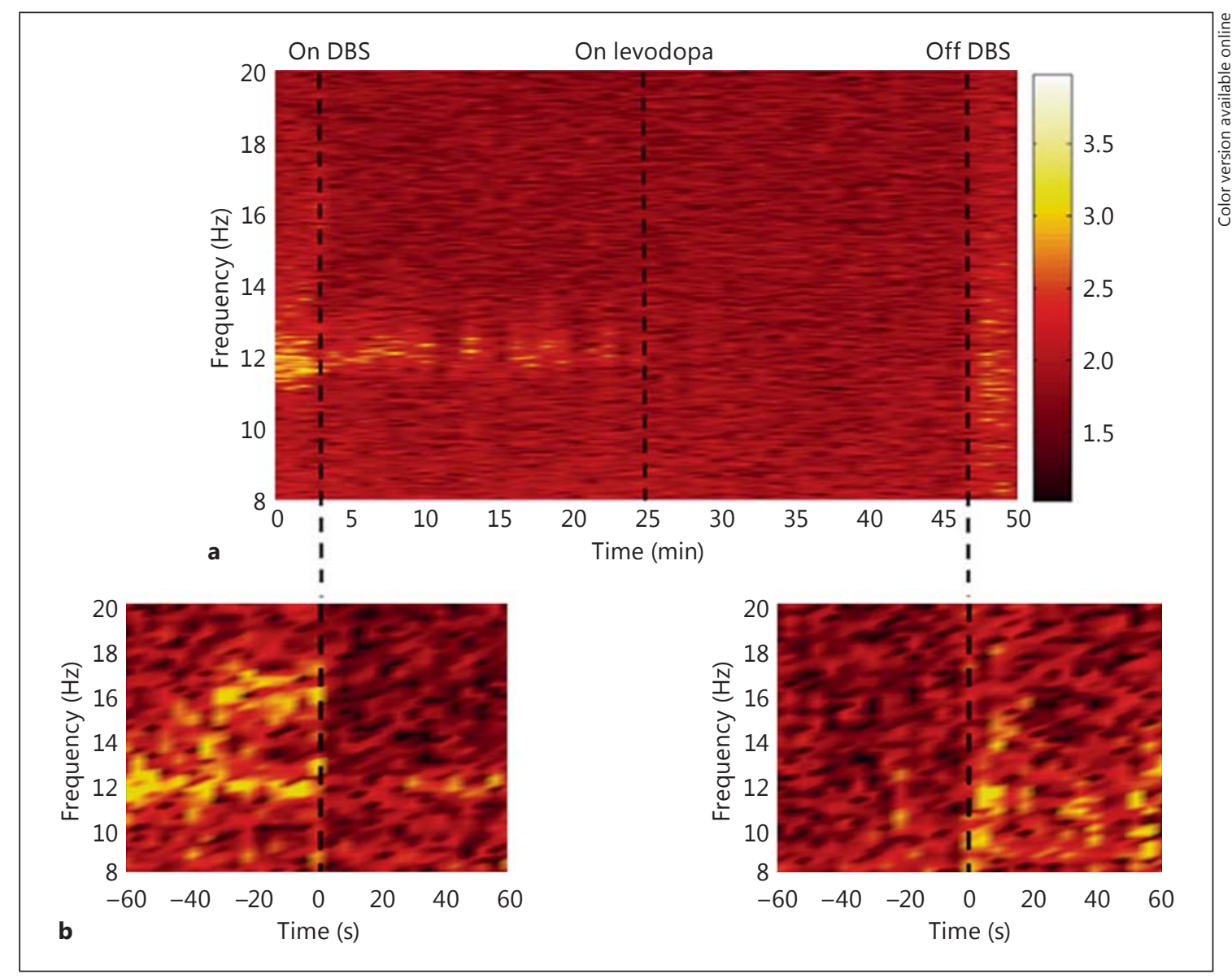

Fig. 2. a Time-frequency plot of beta-frequency LFPs $(8-20 \mathrm{~Hz})$ recorded from the STN during DBS initiation, levodopa administration and termination of stimulation in a patient with PD. b Enlarged view of the transition from DBS 'off to DBS 'on' (left panel) and vice versa (right panel). DBS initiation is associated with a reduction in the power of beta oscillations detected from a DBS electrode implanted in the STN. The residual elevated beta oscillations are completely abolished by dopamine administration. DBS cessation is associated with an immediate return of beta oscillations. Taken with permission from Foffani et al. [30].
$(20-30 \mathrm{~Hz})$. This is supported by the observation that oscillatory power suppression in response to dopaminergic medication is greater in the low beta frequencies compared to high beta frequencies $[29,37]$. However, an understanding of how these different beta subbands relate to the underlying pathophysiology of PD remains enigmatic.

\section{Gamma and High-Frequency Oscillations Are \\ Prokinetic}

In contrast to beta-band activity, theta- [38], gamma[15] and high-frequency band power have been found to increase following dopaminergic treatment $[39,40]$. Interestingly, when patients are off levodopa, gamma activity nonetheless increases bilaterally during active move- ment. In the on state, this increase in gamma-band activity becomes lateralized to the hemisphere contralateral to the active motion [41]. These findings suggest that gamma-band activity may promote normal, voluntary movement. Furthermore, lateralization of the ERS suggests that dopaminergic medication restores this activity to a more normal physiological pattern. Interestingly, highfrequency oscillations (HFO) above $200 \mathrm{~Hz}$ have been detected in the STN of patients undergoing DBS for dystonia and ET - both of which are considered hyperkinetic movement disorders [42]. Furthermore, dopaminergic medication may enhance HFO power while shifting the peak frequency $[29,39,43]$. Specifically, an increase in oscillatory power in the frequency range of $300-350 \mathrm{~Hz}$ has been observed at the expense of $250-\mathrm{Hz}$ oscillations. 
Moreover, the pathophysiological importance of HFO is emphasized by the observation of coupling between beta oscillations and HFO in PD patients. This beta-HFO coupling has been noted to be significantly attenuated following the administration of dopaminergic medication and in patients with less severe PD $[29,43]$. Thus, gammaand high-frequency band activity is generally considered to play an important prokinetic role in the motor symptoms of PD.

\section{Dyskinesia}

Dopaminergic medications have proven effective in treating rigidity, bradykinesia and, to a lesser extent, resting tremor associated with PD. However, prolonged usage in the majority of patients leads to the development of dyskinesia [44]. Levodopa-induced dyskinesia has been correlated with LFP power in PD patients implanted with macroelectrodes in the GPi and/or STN. Consistent with the view of beta power as being antikinetic, recordings from GPi have demonstrated that desynchronization events in the beta-frequency range are significantly associated with dyskinetic states [45]. Additionally, high beta frequencies have been shown to synchronize between the GPi and the STN in a patient bilaterally implanted with macroelectrodes [30].

\section{Resting Tremor}

In contrast to antikinetic symptoms, most studies have indicated that Parkinson's resting tremor does not correlate with beta-frequency oscillations [20,46]. However, epochs in which significant rest tremor is present have been associated with greater power in the low gammafrequency band $(35-55 \mathrm{~Hz})$ which, as described above, is generally viewed as being prokinetic [47]. Additionally, oscillations around tremor frequency $(4.5-5.5 \mathrm{~Hz})$ and double-tremor frequency (approx. $10 \mathrm{~Hz}$ ) correlate with tremor-related electromyogram (EMG) activity. These LFP oscillations and their coherence with tremor appear to be spatially localized in clusters within STN and, to a lesser extent, the zona incerta [48]. Interestingly, the location of tremor-EMG coherence clusters within the STN differs between the resting and postural tremors associated with PD [49]. Furthermore, tremor-related activity is more easily identified with LFP recorded from the STN than from the GPi [50].

The coherence between tremor and LFP oscillations at double the frequency of tremor may represent a real physiological correlate, harmonic waveform noise or a combination of both [51]. However, when comparing tremorrelated EMG-LFP coherence between tremor dominant and bradykinetic PD subtypes, the differences in coherence in the single tremor frequency range between $\mathrm{PD}$ subtypes appeared to be independent of differences in the double-tremor range [49]. This supports the hypothesis that double-tremor frequency LFP is physiologically important.

\section{Postural Instability}

While STN- and GPi-directed DBS have been effective in alleviating tremor, rigidity and bradykinesia, they are ineffective in treating the gait abnormalities and postural instability that are frequently present in PD patients. Furthermore, gait and postural symptoms do not respond to dopaminergic therapy, indicating that they are controlled by a neural pathway that is separate from the dopaminedependent pathway that underlies the tremulous/antikinetic symptoms of PD [52]. Nonhuman primate studies have indicated that the PPN is implicated in the induction and maintenance of locomotion [53]. Building on this work, stimulation of the PPN has also been investigated in the treatment of $\mathrm{PD}[3,4]$.

LFP recordings from DBS macroelectrodes implanted in the PPN have demonstrated that the power of oscillations in the frequency range of $7-11 \mathrm{~Hz}$ is enhanced by the administration of dopaminergic medication and during the planning of self-paced movements. Furthermore, levodopa administration was found to induce synchronization between LFPs of 7-11 Hz and simultaneously recorded electroencephalogram activity [54]. A beta-band peak has also been reported in PPN recordings from $\mathrm{PD}$ patients in the off state $[55,56]$. Relative power in the beta- and sub-beta-frequency bands has been shown to vary spatially and between subjects [56]. In a gait performance paradigm, oscillations of $7-10 \mathrm{~Hz}$ recorded in the PPN - in contrast to beta-band oscillations - significantly correlated with improved gait performance [56].

\section{LFP and Essential Tremor}

DBS targeted at the ventral intermediate nucleus of the thalamus for the treatment of tremor was the first application of DBS approved by the US Food and Drug Administration, and remains the second most common indication of DBS today [57]. Efforts to correlate LFP with tremor have been complicated by the 'microthalamotomy', or 'microlesion' effect that is often observed during surgery for the treatment of ET [58]. Despite this challenge, oscillations of $8-27 \mathrm{~Hz}$ recorded postoperatively from macroelectrodes are significantly coherent with 
tremor frequency recorded from surface EMG of the first dorsal interosseous muscles $[58,59]$.

Additionally, coherence between spatially close $(1 \mathrm{~mm}$ apart), independent thalamic microelectrodes has been compared between ET patients and patients undergoing DBS for the treatment of tremor of multiple sclerosis or for chronic central pain. ET patients, in contrast to subjects with MS tremor or central pain, exhibited enhanced coherence of $5-15 \mathrm{~Hz}$ when the electrodes were located in the ventral intermediate and ventral oral posterior nucleus of the thalamus. In the ventral caudal nucleus, significantly elevated coherence was observed only at 10-15 $\mathrm{Hz}$ [59]. Therefore, these analyses demonstrate the following points: (1) LFP recordings provide information about the spatial heterogeneity of the thalamus, which could help with localization during surgery and (2) differences in the spatial synchronization of LFP may be associated with the pathology of ET.

\section{LFP and Dystonia}

Dystonia is a movement disorder characterized by sustained cocontraction of opposing muscles and is associated with significant disability and pain [60]. Symptoms can be generalized or anatomically confined and are caused by genetic mutation, CNS injury or other disease states [61]. Due to the high prevalence of medication-refractory cases, the FDA approved DBS for the treatment of segmental and generalized dystonia under a humanitarian device exemption status in 2003. While this approval allows for the targeting of either the STN or the $\mathrm{GPi}$, the GPi is the target of choice for DBS in dystonia [62].

The frequency spectrum of LFPs recorded from microelectrodes implanted in the GPi for the treatment of dystonia has been documented in several studies $[63,64]$. These studies have consistently reported relatively high power in the 3- to $12-\mathrm{Hz}$ frequency range compared to other frequency bands. In contrast to recordings from GPe, LFPs recorded from GPi have been shown to synchronize with simultaneously recorded spike activity [63]. Additionally, LFP activity in the frequency ranges of $4-10,11-30$ and $65-85 \mathrm{~Hz}$ significantly correlates with the sternocleidomastoid muscle EMG signal in patients with cervical dystonia [65]. Similarly, in patients with myoclonus dystonia, LFP oscillations of 3-15 Hz are coherent with surface EMG activity associated with affected muscle groups $[64,66]$. This coherence was found to be stronger during the preparation and execution of a wrist extension task [64]. Interestingly, the response to voluntary movement across the LFP spectrum recorded from the GPi in dystonia patients is similar to the response observed in the LFP recordings from the STN in PD patients. During a wrist movement task, a significant ERD is observed at low beta frequencies (approx. 10-24 Hz), while a significant ERS is observed at gamma frequencies (approx. 64-68 Hz) [67].

Patients with cervical dystonia are capable of alleviating dystonic symptoms by touching their chin with the hand that is contralateral to the dystonic head motion [68]. Interestingly, the use of this sensory trick in patients with implanted DBS devices leads to a significant, bilateral desynchronization in the 6 - to $8-\mathrm{Hz}$ frequency range [69]. Furthermore, performing a task similar to the sensory trick (but ineffective in improving dystonic symptoms) leads to a bilateral synchronization in the 4- to $6-\mathrm{Hz}$ frequency range. This finding supports the notion that low-frequency oscillations are implicated in the pathophysiology of dystonia.

The power across the LFP frequency spectrum has been compared between patients with dystonia and $\mathrm{PD}$ implanted with GPi DBS electrodes. Notably, whereas elevated relative power of the beta spectrum has been found in PD, beta power was diminished in dystonia. Furthermore, relative power in the $4-$ to $10-\mathrm{Hz}$ frequency band is higher in dystonia compared to that reported in PD patients in both the 'on' and 'off medication state [18].

Furthermore, Weinberger et al. [70] compared GPi LFP recordings from dystonia and $\mathrm{PD}$ patients. These investigators similarly found higher LFP power in a lower $(8-20 \mathrm{~Hz})$ frequency band compared to PD patients $(11-$ $30 \mathrm{~Hz}$ ). Additionally, they noted less coherence between single-unit activity and LFP oscillations in dystonia compared to PD patients. Therefore, the synchronization between neurons firing at beta frequencies appears to be a more prominent feature of PD than dystonia.

\section{Current and Prospective Clinical Applications of LFP Recording}

\section{Target Localization}

One challenge in DBS surgery remains the ability to precisely place the electrode contacts within the structure of interest - which is often $2-6 \mathrm{~mm}$ in maximal diameter and situated many centimeters from the cortical entry site. Interpatient anatomical variation and insufficient image resolution make it necessary for surgeons to perform neurophysiological recordings with microelec- 
trodes. However, with microelectrodes, the clinician must rely on single neuron activity parameters to interpret the location of the electrode in the brain. These parameters are susceptible to technical (e.g. impedance) and physiological (e.g. cerebrospinal fluid and blood) fluctuations. The subjective nature of interpreting microelectrode signals can lead to variability in correlating single-unit activity with brain region localization. In contrast, because LFP signals reflect an aggregate of electrical activity from an area of neural tissue, they are more resistant to physiological fluctuations. In addition, since LFPs are less variable across conditions, analytic tools that have been developed to correlate LFP activity with electrode depth could be more readily compared across practitioners and institutions and permit standardization of data analysis and interpretation [71]. Specifically, analysis of LFP beta oscillations have been used to delimit the boundaries of the STN [72-74]. In particular, oscillatory beta power is significantly higher within the STN compared to regions rostral and caudal to the STN $[27,75,76]$.

While it is clear that beta activity is enhanced upon entering this region, the STN is a functionally heterogeneous nucleus with a sensorimotor territory situated dorsolaterally and a limbic/associative territory situated ventromedially. Indeed, stimulation of the dorsolateral STN results in optimal symptomatic improvement with minimal stimulation-related side effects compared to ventromedial stimulation [77]. LFP power in the beta-frequency band has been shown to correlate with topographical subregions of the STN and with single-unit activity simultaneously recorded from the STN [6]. Additionally, in a study evaluating the coherence between tremor-related EMG and LFP, the spatial distribution of 'coherence clusters' significantly correlated with the position of the contact that was subsequently selected for chronic stimulation [48]. This EMG activity was coherent with LFP in tremor and double-tremor frequency ranges. Collectively, this evidence suggests that oscillatory activity recorded from the basal ganglia can help inform DBS electrode targeting. Using LFP signals from multiple microelectrodes has the advantage of standardizing the interpretation of STN subdivisional boundaries. LFPs will not replace the need to use multiple electrodes, given the spatial area that the microelectrode is intended to resolve from imaging variability and error. However, real-time analysis of LFP signals may be more instructive and objective for the placement of the DBS implant and ultimately might serve as the better adjunct to intraoperative MRI placement of DBS electrodes.

Clinical Correlations and Implications of LFPs

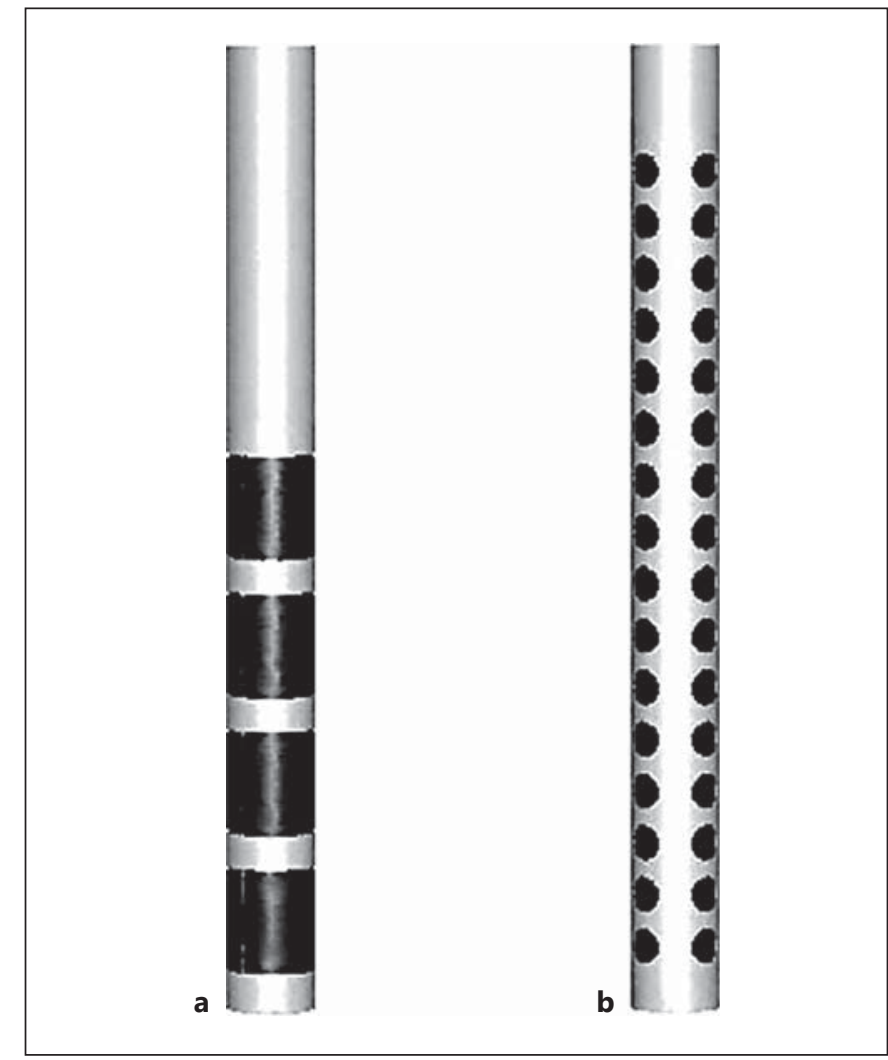

Fig. 3. a Contemporary DBS macroelectrode with 4 cylindrical contacts. b Novel high spatial resolution electrode with 64 contacts. Taken with permission from Zaidel et al. [74].

\section{Programming Guidance}

The programming of DBS devices is currently achieved via a lengthy algorithmic process in which each of the four contacts is tested individually while increasing the voltage or current. The clinician makes note of benefits and side effects - both objective and through patient self-report and ultimately selects the optimal settings for chronic stimulation. While this process is uncomfortable for patients (who must be off medication during the process) and laborious for both clinicians and patients alike, it remains feasible due to relatively small combinations of programming parameters. However, the next generation of DBS macroelectrodes is likely to present increased complexity for programming. For instance, Martens et al. [78] have developed a 64-contact macroelectrode that could allow for the 'steering' of current away from structures such as the internal capsule - the stimulation of which would result in side effects (fig. 3). Such guided stimulation is achieved by using multiple, smaller electrodes arrayed around the circumference of a solid sup- 


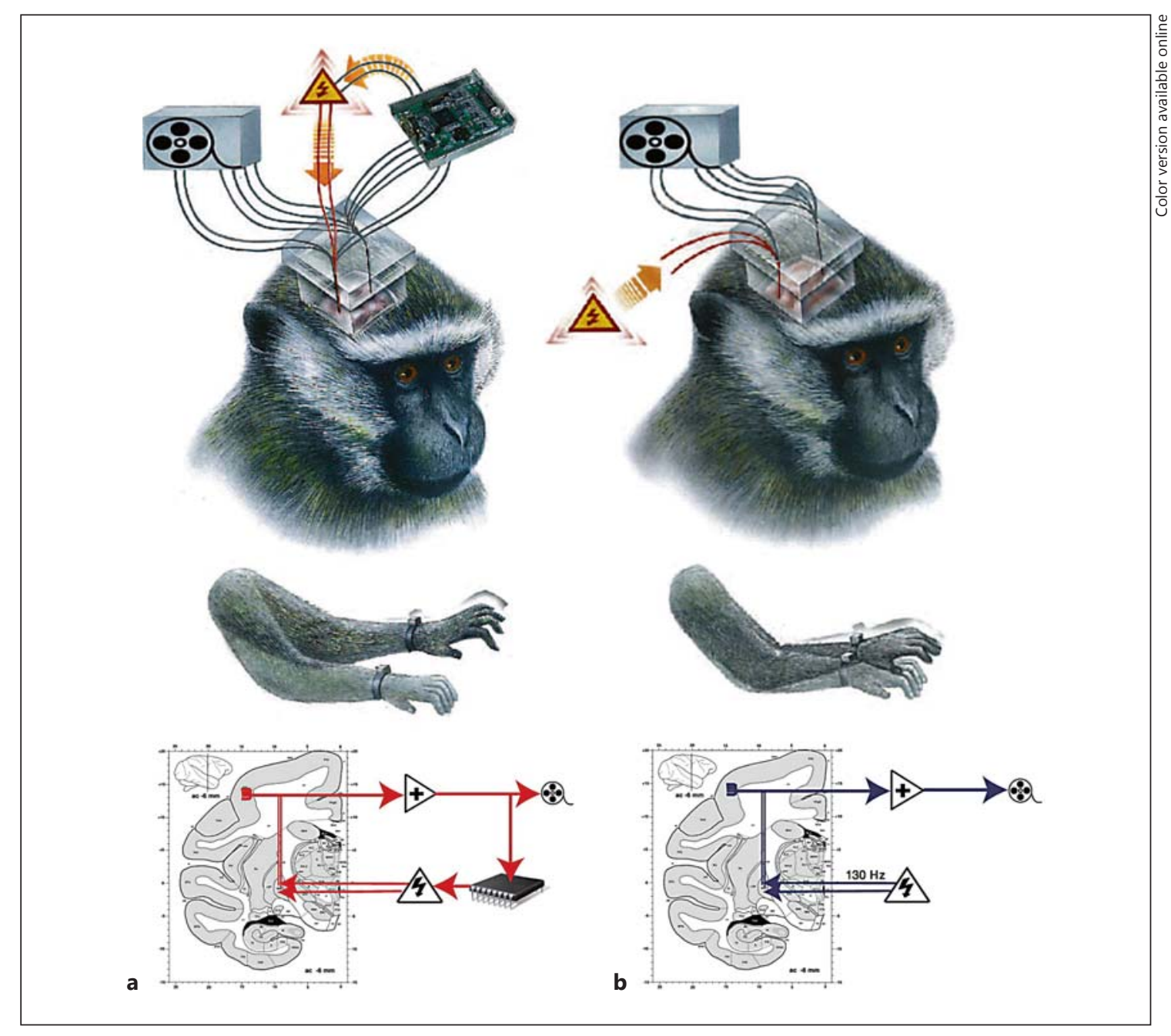

Fig. 4. a Schematic depiction of the African green monkey closed-loop stimulation paradigm in Rosin et al. [89]. Single-unit recordings were detected from 6 electrodes (2 GPi, 4 M1) by a data acquisition system and digital signal-processing chip. Detection of a neuronal spike triggered a short-train stimulus. $\mathbf{b}$ Schematic depiction of an open-loop stimulation paradigm in an African green monkey.

port instead of the cylindrical contacts used on contemporary electrodes. Although the presence of 64 contacts would render current programming methods impractical, novel analytic methods based on LFPs have been developed to assess spatial reach and efficacy of DBS stimulation [79]. The use of LFPs in improving DBS clinical outcomes has largely been explored in the setting of DBS implantation into STN for the treatment of PD [30, 35, 80, 81]. Recent work in STN LFP analysis suggests that programming could be improved through analysis of macroelectrode-recorded LFPs and modeling of stimulation parameters $[79,82,83]$. One direct clinical application relates to the chronic hypersynchrony evidenced in the basal ganglia of Parkinson's patients: better clinical outcomes correlate with the degree of beta hypersynchrony. Analysis of LFPs through the macroelectrode could be used to set optimal clinical stimulation parameters. Interestingly, those contacts associated with optimal stimulation efficacy significantly correlate with contacts exhibiting maximal beta and gamma power [26, 27, 84]. Moreover, chronic stimulation through contacts which are further from the apparent source of beta activity is associated with a poorer response to stimulation [26]. Thus, LFPs recorded from implanted DBS electrodes might prove useful in improving and automating the programming process. One specific advantage of a DBS electrode 


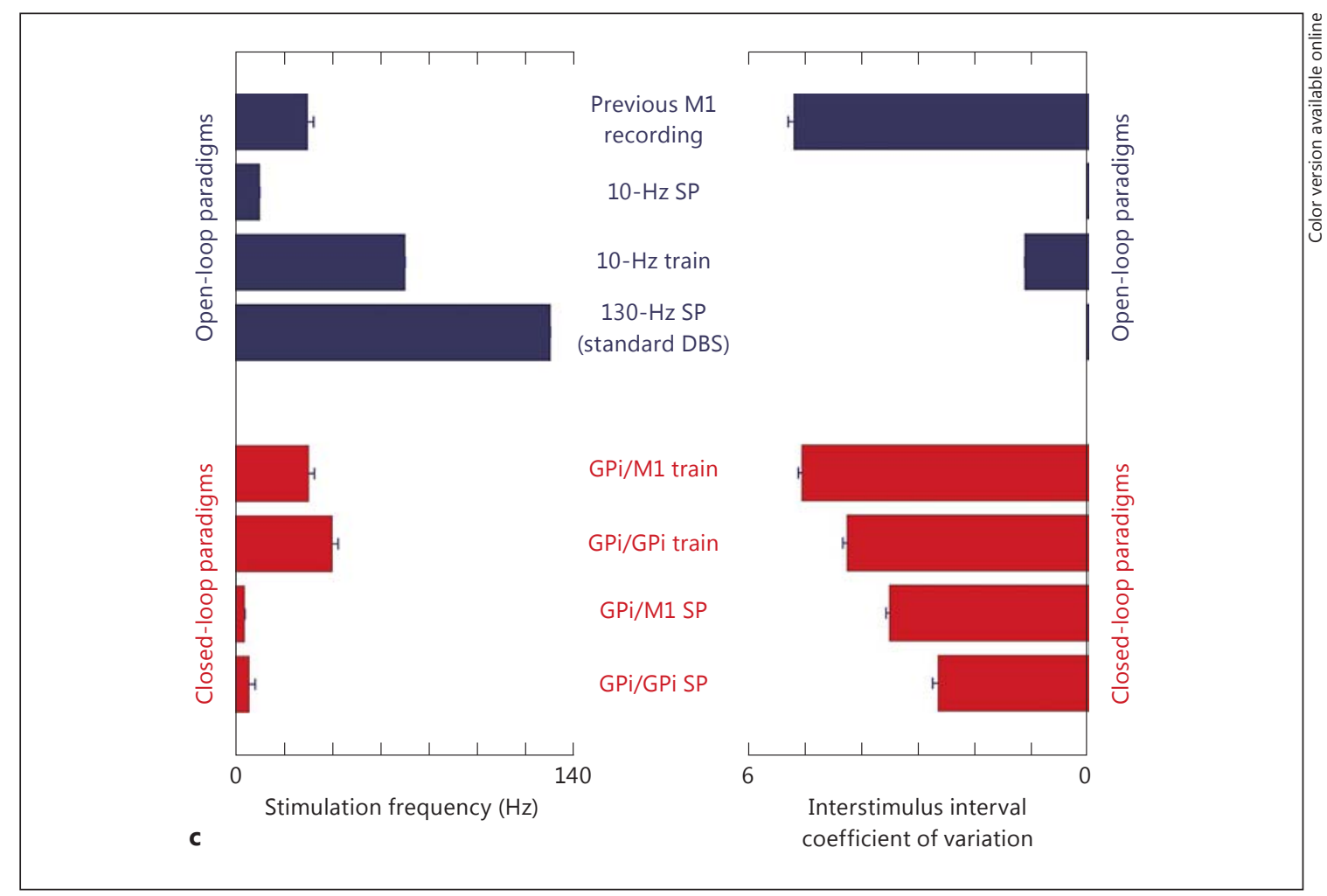

Fig. 4. $c$ The stimulation frequency and coefficient of variation of the interstimulus intervals for the various stimulation paradigms. SP = Stimulation paradigm. Taken with permission from Levy et al. [80].

design that has denser, smaller diameter contacts would be the selective distribution of electrical activity patterns ('steering current'). Furthermore, activity patterns could be based on LFP information, which may reduce the overall stimulation output, thus increasing battery life.

\section{Closed-Loop Therapies}

Another challenge in treating movement disorders with DBS is the inability of existing devices to do the following: (1) monitor patient symptoms and (2) adjust stimulation parameters accordingly. The stimulation parameters (voltage, current, pulse width, polarity of stimulation and contact selection) can only be modified by programming personnel using an external computer wand placed over the patient's implanted pulse generator. This process requires patients to visit a clinic staffed with personnel who have expertise in programming implanted DBS devices, thereby inconveniencing patients and burdening these clinics with repeated appointments. Furthermore, without a sensing component to the existing DBS devices, there is no feedback mechanism to allow the device to be turned off during sleep, when Parkinson's symptoms disappear, or to be ramped up when symptoms increase between medication doses. Therefore, there is much interest in developing a closed-loop therapy in which a relevant neural marker, such as LFP oscillations, provides feedback that directs the modulation of stimulation parameters in real time [85-88].

Rosin et al. [89] investigated the feasibility of such a closed-loop stimulating device in an MPTP primate model of PD. Single-unit recordings from the primary motor cortex (M1) and the GPi were used to direct the stimulation of the GPi. Specifically, the detection of a spike in either the GPi or the M1 triggered a short-train stimulus (7 pulses at $130 \mathrm{~Hz}, 80-\mathrm{ms}$ latency). This form of closedloop stimulation proved superior to continuous, GPi-targeted stimulation in suppressing pallidal spike and oscillatory activity. Furthermore, closed-loop stimulation was associated with a greater reduction in akinesia compared to continuous stimulation (fig. 4).

Currently, the battery life of nonrechargeable DBS generators is approximately 3-5 years, depending on the stimulation parameters used. Generator replacement surgery involves an invasive 15 - to 20 -min procedure per- 
formed under monitored anesthesia care. Replacement surgeries are associated with a small but significant risk of infection and damage to the existing device. Thus, strategies to increase the battery life of DBS devices are important, considering the number of battery replacement surgeries required over the lifetime of, for instance, a 40-yearold patient with ET. If a closed-loop device was capable of identifying when a patient was asleep, stimulation could be turned off during this period, thus increasing the battery life of the device. In fact, beta-band LFP power has been reported to be significantly lower during stages 2 and 4 of sleep compared to when patients are awake [90]. It is not practical to require PD patients to self-regulate their implantable pulse generator power for sleeping and waking, as a significant number of patients with PD often experience neurogenic bladder dysfunction, which is associated with a greater frequency of micturition [91]. Intentional tremor, a primary sequela of $\mathrm{PD}$, would make turning the implantable pulse generator back on (for bladder evacuation or upon waking) difficult if not impossible. Additionally, different motor symptoms may be determined to have unique LFP profiles, which could be ameliorated with a specific combination of stimulation parameters or by switching active contacts. For instance, differences in stimulation efficacy for bradykinesia have been observed to depend on the site of stimulation within the STN. Switching off electrodes placed in the lateral STN resulted in a rapid return of bradykinesia; however, a more medial placement resulted in a slower return of bradykinesia [92]. Such stimulation device improvements could increase the time between battery changes.
Another consideration for developing a closed-loop DBS device is the integrity of the electrode-brain interface. Postmortem histopathological studies have found the DBS electrode in the brain to be encapsulated by a thin, GFAP-positive capsule years after initial implantation $[93,94]$. Moreover, a recent study showed that in patients with PD, the LFP power in the beta-frequency band was significantly lower 3-7 years after the initial DBS implant compared to power recorded at the time of DBS surgery [95]. In contrast, the magnitude of the movement-related desynchronization in the beta band was preserved and detectable over time [95]. Therefore, despite the decrease in beta power over time, the ERD preservation supports the feasibility of using beta-band activity to inform a closed-loop device.

\section{Summary}

LFPs recorded from implanted DBS electrodes in human subjects have provided researchers and clinicians with a novel method for understanding, and potentially refining treatments for movement disorders. Correlating aspects of the LFP frequency spectrum with clinical symptoms has provided new insights into the pathophysiology of these disorders, and mounting evidence suggests that LFPs will be useful in improving current therapies in this arena. In particular, LFP oscillations have proven to be useful in localizing DBS surgical targets. LFPs may ultimately be able to inform a closed-loop DBS device that is responsive to individual patient symptoms in real time.

\section{References}

-1 Einevoll GT, Kayser C, Logothetis NK, Panzeri S: Modelling and analysis of local field potentials for studying the function of cortical circuits. Nat Rev Neurosci 2013;14:770-785.

$>2$ Brown P: Oscillatory nature of human basal ganglia activity: relationship to the pathophysiology of Parkinson's disease. Mov Disord 2003; 18:357-363.

-3 Jenkinson N, Nandi D, Aziz TZ, Stein JF: Pedunculopontine nucleus: a new target for deep brain stimulation for akinesia. Neuroreport 2005;16:1875-1876.

4 Mazzone P, Sposato S, Insola A, Scarnati E: The deep brain stimulation of the pedunculopontine tegmental nucleus: towards a new stereotactic neurosurgery. J Neural Transm 2011;118:1431-1451.
5 Brown P: Oscillatory nature of human basal ganglia activity: relationship to the pathophysiology of Parkinson's disease. Mov Disord 2003;18:357-363.

-6 Kuhn AA, Trottenberg T, Kivi A, Kupsch A, Schneider GH, Brown P: The relationship between local field potential and neuronal discharge in the subthalamic nucleus of patients with Parkinson's disease. Exp Neurol 2005; 194:212-220.

7 Pfurtscheller G, Lopez da Silva FH: Event-related EEG/MEG synchronization and desynchronization: basic principles. Clin Neurophysiol 1999;110:1842-1857.

8 Pfurtscheller G: Event-related desynchronization; in Pfurtscheller G, Lopes da Silva FH (eds): Handbook of Electroencephalography and Clinical Neurophysiology, rev ed. Amsterdam, Elsevier Science, 1999.
>9 Lopes da Silva FH: Event-related neural activities: what about phase? Prog Brain Res 2006;159:3-17.

-10 Langston JW, Forno LS, Rebert CS, Irwin I: Selective nigral toxicity after systemic administration of 1-methyl-4-phenyl-1,2,5,6-tetrahydropyrine (MPTP) in the squirrel monkey. Brain Res 1984;292:390-394.

-11 Burns RS, Chiueh CC, Markey SP, Ebert MH, Jacobowitz DM, Kopin IJ: A primate model of parkinsonism: selective destruction of dopaminergic neurons in the pars compacta of the substantia nigra by N-methyl-4-phenyl1,2,3,6-tetrahydropyridine. Proc Natl Acad Sci USA 1983;80:4546-4550.

$>12$ DeLong MR: Primate models of movement disorders of basal ganglia origin. Trends Neurosci 1990;13:281-285. 
$\checkmark 13$ Bergman H, Wichmann T, Karmon B, DeLong MR: The primate subthalamic nucleus. II. Neuronal activity in the MPTP model of parkinsonism. J Neurophysiol 1994;72:507520.

- 14 Nini A, Feingold A, Slovin H, Bergman H: Neurons in the globus pallidus do not show correlated activity in the normal monkey, but phase-locked oscillations appear in the MPTP model of parkinsonism. J Neurophysiol 1995; 74:1800-1805.

- 15 Brown P, Oliviero A, Mazzone P, Insola A, Tonali P, Di Lazzaro V: Dopamine dependency of oscillations between subthalamic nucleus and pallidum in Parkinson's disease. J Neurosci 2001;21:1033-1038.

- 16 Alegre M, Alonso-Frech F, Rodriguez-Oroz MC, Guridi J, Zamarbide I, Valencia M, Manrique $\mathrm{M}$, Obeso JA, Artieda J: Movement-related changes in oscillatory activity in the human subthalamic nucleus: ipsilateral versus contralateral movements. Eur J Neurosci 2005;22:2315-2324.

-17 Cassidy M, Mazzone P, Oliviero A, Insola A, Tonali P, Di Lazzaro V, Brown P: Movementrelated changes in synchronization in the human basal ganglia. Brain 2002;125:12351246.

18 Silberstein P, Kuhn AA, Kupsch A, Trottenberg T, Krauss JK, Wohrle JC, Mazzone P, Insola A, Di Lazzaro V, Oliviero A, Aziz T, Brown P: Patterning of globus pallidus local field potentials differs between Parkinson's disease and dystonia. Brain 2003;126:25972608.

19 Weinberger M, Dostrovsky JO: A basis for the pathological oscillations in basal ganglia: the crucial role of dopamine. Neuroreport 2011 22:151-156.

-20 Ray NJ, Jenkinson N, Wang S, Holland P, Brittain JS, Joint C, Stein JF, Aziz T: Local field potential beta activity in the subthalamic nucleus of patients with Parkinson's disease is associated with improvements in bradykinesia after dopamine and deep brain stimulation. Exp Neurol 2008;213:108-113.

21 Weinberger M, Mahant N, Hutchison WD, Lozano AM, Moro E, Hodaie M, Lang AE, Dostrovsky JO: Beta oscillatory activity in the subthalamic nucleus and its relation to dopaminergic response in Parkinson's disease. J Neurophysiol 2006;96:3248-3256.

22 Kuhn AA, Tsui A, Aziz T, Ray N, Brucke C, Kupsch A, Schneider GH, Brown P: Pathological synchronisation in the subthalamic nucleus of patients with Parkinson's disease relates to both bradykinesia and rigidity. Exp Neurol 2009;215:380-387.

-23 Kuhn AA, Brucke C, Schneider GH, Trottenberg T, Kivi A, Kupsch A, Capelle HH, Krauss JK, Brown P: Increased beta activity in dystonia patients after drug-induced dopamine deficiency. Exp Neurol 2008;214:140-143.
24 Weinberger MMN, Hutchison WD, Lozano AM, Moro E, Hodaie M, Lang AE, Dostrovsky JO: Beta oscillatory activity in the subthalamic nucleus and its relation to dopaminergic response in Parkinson's disease. J Neurophysiol 2006;96:3248-3256.

25 Pogosyan A, Yoshida F, Chen CC, MartinezTorres I, Foltynie T, Limousin P, Zrinzo L, Hariz MI, Brown P: Parkinsonian impairment correlates with spatially extensive subthalamic oscillatory synchronization. Neuroscience 2010;171:245-257.

26 Yoshida F, Martinez-Torres I, Pogosyan A, Holl E, Petersen E, Chen CC, Foltynie T, Limousin P, Zrinzo LU, Hariz MI, Brown P: Value of subthalamic nucleus local field potentials recordings in predicting stimulation parameters for deep brain stimulation in Parkinson's disease. J Neurol Neurosurg Psychiatry 2010;81:885-889.

27 Holdefer RN, Cohen BA, Greene KA: Intraoperative local field recording for deep brain stimulation in Parkinson's disease and essential tremor. Mov Disord 2010;25:2067-2075.

28 Chen CC, Hsu YT, Chan HL, Chiou SM, Tu PH, Lee ST, Tsai CH, Lu CS, Brown P: Complexity of subthalamic $13-35 \mathrm{~Hz}$ oscillatory activity directly correlates with clinical impairment in patients with Parkinson's disease. Exp Neurol 2010;224:234-240.

29 Lopez-Azcarate J, Tainta M, Rodriguez-Oroz MC, Valencia M, Gonzalez R, Guridi J, Iriarte J, Obeso JA, Artieda J, Alegre M: Coupling between beta and high-frequency activity in the human subthalamic nucleus may be a pathophysiological mechanism in Parkinson's disease. J Neurosci 2010;30:6667-6677.

-30 Foffani G, Ardolino G, Egidi M, Caputo E, Bossi B, Priori A: Subthalamic oscillatory activities at beta or higher frequency do not change after high-frequency DBS in Parkinson's disease. Brain Res Bull 2006;69:123130.

31 Rossi L, Foffani G, Marceglia S, Bracchi F, Barbieri S, Priori A: An electronic device for artefact suppression in human local field potential recordings during deep brain stimulation. J Neural Eng 2007;4:96-106.

32 Giannicola G, Marceglia S, Rossi L, MrakicSposta S, Rampini P, Tamma F, Cogiamanian F, Barbieri S, Priori A: The effects of levodopa and ongoing deep brain stimulation on subthalamic beta oscillations in Parkinson's disease. Exp Neurol 2010;226:120-127.

33 Doyle LM, Kuhn AA, Hariz M, Kupsch A, Schneider GH, Brown P: Levodopa-induced modulation of subthalamic beta oscillations during self-paced movements in patients with Parkinson's disease. Eur J Neurosci 2005;21: 1403-1412.

34 Alegre M, Rodriguez-Oroz MC, Valencia M, Perez-Alcazar M, Guridi J, Iriarte J, Obeso JA, Artieda J: Changes in subthalamic activity during movement observation in Parkinson's disease: is the mirror system mirrored in the basal ganglia? Clin Neurophysiol 2010;121: 414-425.
35 Hsu YT, Lai HY, Chang YC, Chiou SM, Lu MK, Lin YC, Liu YL, Chen CC, Huang HC, Chien TF, Lin SZ, Chen YY, Tsai CH: The role of the sub-thalamic nucleus in the preparation of volitional movement termination in Parkinson's disease. Exp Neurol 2012;233: 253-263.

36 Kuhn AA, Kupsch A, Schneider GH, Brown $P$ : Reduction in subthalamic $8-35 \mathrm{~Hz}$ oscillatory activity correlates with clinical improvement in Parkinson's disease. Eur J Neurosci 2006;23:1956-1960.

37 Priori A, Foffani G, Pesenti A, Tamma F, Bianchi AM, Pellegrini M, Locatelli M, Moxon KA, Villani RM: Rhythm-specific pharmacological modulation of subthalamic activity in Parkinson's disease. Exp Neurol 2004;189: 369-379.

- 38 Priori A, Foffani G, Pesenti A, Tamma F, Bianchi AM, Pellegrini M, Locatelli M, Moxon KA, Villani RM: Rhythm-specific pharmacological modulation of subthalamic activity in Parkinson's disease. Exp Neurol 2004;189: 369-379.

39 Foffani G, Priori A, Egidi M, Rampini P, Tamma F, Caputo E, Moxon KA, Cerutti S, Barbieri S: $300-\mathrm{Hz}$ subthalamic oscillations in Parkinson's disease. Brain 2003;126:21532163.

40 Kane A, Hutchison WD, Hodaie M, Lozano AM, Dostrovsky JO: Dopamine-dependent high-frequency oscillatory activity in thalamus and subthalamic nucleus of patients with Parkinson's disease. Neuroreport 2009;20: 1549-1553.

-41 Androulidakis AG, Kuhn AA, Chen CC, Blomstedt P, Kempf F, Kupsch A, Schneider GH, Doyle L, Dowsey-Limousin P, Hariz MI, Brown P: Dopaminergic therapy promotes lateralized motor activity in the subthalamic area in Parkinson's disease. Brain 2007;130: 457-468.

42 Danish SF, Moyer JT, Finkel LH, Baltuch GH Jaggi JL, Priori A, Foffani G: High-frequency oscillations $(>200 \mathrm{~Hz})$ in the human non-parkinsonian subthalamic nucleus. Brain Res Bull 2007;74:84-90.

-43 Ozkurt TE, Butz M, Homburger M, Elben S, Vesper J, Wojtecki L, Schnitzler A: High frequency oscillations in the subthalamic nucleus: a neurophysiological marker of the motor state in Parkinson's disease. Exp Neurol 2011; 229:324-331.

44 Calabresi P, Di Filippo M, Ghiglieri V, Tambasco N, Picconi B: Levodopa-induced dyskinesias in patients with Parkinson's disease: filling the bench-to-bedside gap. Lancet Neurol 2010;9:1106-1117.

45 Silberstein P, Oliviero A, Di Lazzaro V, Insola A, Mazzone P, Brown P: Oscillatory pallidal local field potential activity inversely correlates with limb dyskinesias in Parkinson's disease. Exp Neurol 2005;194:523-529. 
-46 Weinberger M, Hutchison WD, Lozano AM, Hodaie M, Dostrovsky JO: Increased gamma oscillatory activity in the subthalamic nucleus during tremor in Parkinson's disease patients. J Neurophysiol 2009;101:789-802.

-47 Weinberger M, Hutchison WD, Lozano AM, Hodaie M, Dostrovsky JO: Increased gamma oscillatory activity in the subthalamic nucleus during tremor in Parkinson's disease patients. J Neurophysiol 2009;101:789-802.

48 Reck C, Florin E, Wojtecki L, Krause H, Groiss S, Voges J, Maarouf M, Sturm V, Schnitzler A, Timmermann L: Characterisation of tremor-associated local field potentials in the subthalamic nucleus in Parkinson's disease. Eur J Neurosci 2009;29:599-612.

-49 Reck C, Himmel M, Florin E, Maarouf M, Sturm V, Wojtecki L, Schnitzler A, Fink GR, Timmermann L: Coherence analysis of local field potentials in the subthalamic nucleus: differences in parkinsonian rest and postural tremor. Eur J Neurosci 2010;32:1202-1214.

50 Burgess JG, Warwick K, Ruiz V, Gasson MN, Aziz TZ, Brittain JS, Stein J: Identifying tremor-related characteristics of basal ganglia nuclei during movement in the Parkinsonian patient. Parkinsonism Relat Disord 2010;16: 671-675.

51 Wang S, Aziz TZ, Stein JF, Bain PG, Liu X: Physiological and harmonic components in neural and muscular coherence in Parkinsonian tremor. Clin Neurophysiol 2006;117: 1487-1498.

52 Hamani C, Stone S, Laxton A, Lozano AM: The pedunculopontine nucleus and movement disorders: anatomy and the role for deep brain stimulation. Parkinsonism Relat Disord 2007;13(suppl 3):S276-S280.

53 Garcia-Rill E: The pedunculopontine nucleus. Prog Neurobiol 1991;36:363-389.

-54 Androulidakis AG, Mazzone P, Litvak V, Penny W, Dileone M, Gaynor LM, Tisch S, Di Lazzaro V, Brown P: Oscillatory activity in the pedunculopontine area of patients with Parkinson's disease. Exp Neurol 2008;211:59-66.

- 55 Tsang EW, Hamani C, Moro E, Mazzella F, Poon YY, Lozano AM, Chen R: Involvement of the human pedunculopontine nucleus region in voluntary movements. Neurology 2010;75:950-959.

56 Thevathasan W, Pogosyan A, Hyam JA, Jenkinson N, Foltynie T, Limousin P, Bogdanovic M, Zrinzo L, Green AL, Aziz TZ, Brown $\mathrm{P}$ : Alpha oscillations in the pedunculopontine nucleus correlate with gait performance in parkinsonism. Brain 2012;135:148-160.

- 57 Deuschl G, Raethjen J, Hellriegel H, Elble R: Treatment of patients with essential tremor. Lancet Neurol 2011;10:148-161.

58 Marsden JF, Ashby P, Limousin-Dowsey P, Rothwell JC, Brown P: Coherence between cerebellar thalamus, cortex and muscle in man: cerebellar thalamus interactions. Brain 2000;123(part 7):1459-1470.
Kane A, Hutchison WD, Hodaie M, Lozano AM, Dostrovsky JO: Enhanced synchronization of thalamic theta band local field poten tials in patients with essential tremor. Exp Neurol 2009;217:171-176.

60 Fahn S: The varied clinical expressions of dystonia. Neurol Clin 1984;2:541-554.

61 Breakefield XO, Blood AJ, Li Y, Hallett M, Hanson PI, Standaert DG: The pathophysiological basis of dystonias. Nat Rev Neurosci 2008;9:222-234.

62 Ostrem JL, Starr PA: Treatment of dystonia with deep brain stimulation. Neurotherapeutics 2008;5:320-330.

63 Chen CC, Kuhn AA, Trottenberg T, Kupsch A, Schneider GH, Brown P: Neuronal activity in globus pallidus interna can be synchronized to local field potential activity over 3-12 $\mathrm{Hz}$ in patients with dystonia. Exp Neurol 2006;202:480-486.

64 Foncke EM, Bour LJ, Speelman JD, Koelman $\mathrm{JH}$, Tijssen MA: Local field potentials and oscillatory activity of the internal globus pallidus in myoclonus-dystonia. Mov Disord 2007;22:369-376.

65 Chen CC, Kuhn AA, Hoffmann KT, Kupsch A, Schneider GH, Trottenberg T, Krauss JK, Wohrle JC, Bardinet E, Yelnik J, Brown P: Oscillatory pallidal local field potential activity correlates with involuntary EMG in dystonia. Neurology 2006;66:418-420.

66 Liu X, Griffin IC, Parkin SG, Miall RC, Rowe JG, Gregory RP, Scott RB, Aziz TZ, Stein JF: Involvement of the medial pallidum in focal myoclonic dystonia: a clinical and neurophysiological case study. Mov Disord 2002; 17:346-353.

-67 Tsang EW, Hamani C, Moro E, Mazzella F, Lozano AM, Hodaie M, Yeh IJ, Chen R: Movement related potentials and oscillatory activities in the human internal globus pallidus during voluntary movements. J Neurol Neurosurg Psychiatry 2012;83:91-97.

68 Schramm A, Reiners K, Naumann M: Complex mechanisms of sensory tricks in cervical dystonia. Mov Disord 2004;19:452-458.

69 Tang JK, Mahant N, Cunic D, Chen R, Moro E, Lang AE, Lozano AM, Hutchison WD, Dostrovsky JO: Changes in cortical and pallidal oscillatory activity during the execution of a sensory trick in patients with cervical dystonia. Exp Neurol 2007;204:845-848.

70 Weinberger M, Hutchison WD, Alavi M, Hodaie M, Lozano AM, Moro E, Dostrovsky JO: Oscillatory activity in the globus pallidus internus: comparison between Parkinson's disease and dystonia. Clin Neurophysiol 2012; 123:358-368.

71 Winestone JS, Zaidel A, Bergman H, Israel Z: The use of macroelectrodes in recording cellular spiking activity. J Neurosci Methods 2012;206:34-39.

-72 Moran A, Bergman H, Israel Z, Bar-Gad I: Subthalamic nucleus functional organization revealed by parkinsonian neuronal oscillations and synchrony. Brain 2008;131:33953409 .
73 Zaidel A, Spivak A, Grieb B, Bergman H, Israel Z: Subthalamic span of beta oscillations predicts deep brain stimulation efficacy for patients with Parkinson's disease. Brain 2010; 133:2007-2021.

74 Zaidel A, Spivak A, Shpigelman L, Bergman $\mathrm{H}$, Israel Z: Delimiting subterritories of the human subthalamic nucleus by means of microelectrode recordings and a Hidden Markov Model. Mov Disord 2009;24:17851793.

75 Trottenberg T, Kupsch A, Schneider GH, Brown P, Kuhn AA: Frequency-dependent distribution of local field potential activity within the subthalamic nucleus in Parkinson's disease. Exp Neurol 2007;205:287291.

76 Chen CC, Pogosyan A, Zrinzo LU, Tisch S Limousin P, Ashkan K, Yousry T, Hariz MI, Brown P: Intra-operative recordings of local field potentials can help localize the subthalamic nucleus in Parkinson's disease surgery. Exp Neurol 2006;198:214-221.

-77 Maks CB, Butson CR, Walter BL, Vitek JL, McIntyre CC: Deep brain stimulation activation volumes and their association with neurophysiological mapping and therapeutic outcomes. J Neurol Neurosurg Psychiatry 2009;80:659-666.

78 Martens HC, Toader E, Decre MM, Anderson DJ, Vetter R, Kipke DR, Baker KB, Johnson MD, Vitek JL: Spatial steering of deep brain stimulation volumes using a novel lead design. Clin Neurophysiol 2011;122:558-566.

79 Lempka SF, McIntyre CC: Theoretical analysis of the local field potential in deep brain stimulation applications. PLoS One 2013; 8:e59839.

80 Levy R, Ashby P, Hutchison WD, Lang AE, Lozano AM, Dostrovsky JO: Dependence of subthalamic nucleus oscillations on movement and dopamine in Parkinson's disease. Brain 2002;125:1196-1209.

-81 Shimamoto SA, Ryapolova-Webb ES, Ostrem JL, Galifianakis NB, Miller KJ, Starr PA: Subthalamic nucleus neurons are synchronized to primary motor cortex local field potentials in Parkinson's disease. J Neurosci 2013;33: 7220-7233.

82 Chaturvedi A, Lujan JL, McIntyre CC: Artificial neural network based characterization of the volume of tissue activated during deep brain stimulation. J Neural Eng 2013;10: 056023.

83 Leski S, Linden H, Tetzlaff T, Pettersen KH, Einevoll GT: Frequency dependence of signal power and spatial reach of the local field potential. PLoS Comput Biol 2013;9:e1003137.

84 Ince NF, Gupte A, Wichmann T, Ashe J, Henry T, Bebler M, Eberly L, Abosch A: Selection of optimal programming contacts based on local field potential recordings from subthalamic nucleus in patients with Parkinson's disease. Neurosurgery 2010;67:390-397. 
85 Kent AR, Grill WM: Instrumentation to record evoked potentials for closed-loop control of deep brain stimulation. Conf Proc IEEE Eng Med Biol Soc 2011;2011:67776780.

86 Eberle W, Penders J, Yazicioglu RF: Closing the loop for deep brain stimulation implants enables personalized healthcare for Parkinson's disease patients. Conf Proc IEEE Eng Med Biol Soc 2011;2011:1556-1558.

87 Boraud T: Closed-loop stimulation: the future of surgical therapy of brain disorders? Mov Disord 2012;27:200.

88 Afshar P, Khambhati A, Stanslaski S, Carlson $D$, Jensen R, Linde D, Dani S, Lazarewicz M, Cong P, Giftakis J, Stypulkowski P, Denison T: A translational platform for prototyping closed-loop neuromodulation systems. Front Neural Circuits 2013;6:117.
89 Rosin B, Slovik M, Mitelman R, Rivlin-Etzion M, Haber SN, Israel Z, Vaadia E, Bergman H: Closed-loop deep brain stimulation is superior in ameliorating parkinsonism. Neuron 2011;72:370-384.

90 Urrestarazu E, Iriarte J, Alegre M, Clavero P, Rodriguez-Oroz MC, Guridi J, Obeso JA, Artieda J: Beta activity in the subthalamic nucleus during sleep in patients with Parkinson's disease. Mov Disord 2009;24:254-260.

91 Dorsher PT, McIntosh PM: Neurogenic bladder. Adv Urol 2012;2012:816274.

92 Cooper SE, Noecker AM, Abboud H, Vitek JL, McIntyre CC: Return of bradykinesia after subthalamic stimulation ceases: relationship to electrode location. Exp Neurol 2011;231: 207-213.
93 Sun DA, Yu H, Spooner J, Tatsas AD, Davis T, Abel TW, Kao C, Konrad PE: Postmortem analysis following 71 months of deep brain stimulation of the subthalamic nucleus for Parkinson disease. J Neurosurg 2008; 109: 325-329.

94 Nielsen MS, Bjarkam CR, Sorensen JC, Bojsen-Moller M, Sunde NA, Ostergaard K: Chronic subthalamic high-frequency deep brain stimulation in Parkinson's disease - a histopathological study. Eur J Neurol 2007; 14:132-138.

95 Abosch A, Lanctin D, Onaran I, Eberly L, Spaniol M, Ince NF: Long-term recordings of local field potentials from implanted deep brain stimulation electrodes. Neurosurgery 2012;71:804-814. 